\title{
XENODIAGNÓSTICO ARTIFICIAL “POST-MORTEM" EM CHAGÁSICOS CRÔNICOS
}

\section{Edison Reis Lopes 1, Edmundo Chapadeiro 1, Zigman Brener2, José Umberto Franciscon', Jarbas E. Cardoso2, Sheila J. Adad1 e Sebastião Tostes Júnior1.}

\begin{abstract}
A fim de obter metodologias que permitam estabelecer, com segurança, o diagnóstico "post-mortem" da infecção chagásica, adaptou-se o xenodiagnóstico artificial a necropsiados com diferentes tempos de óbito. O teste foi positivo em três(30\%) de dez chagásicos autopsiados. $O$ tempo decorrido entre o êxito letal e o início do repasto pelos triatomíneos destes chagásicos foi de duas horas, duas horas e quinze minutos $e$ sete horas, respectivamente.

Discutem-se os fatores que podem explicar a sobrevivência do Trypanosoma cruzi no hospedeiro morto bem como as aplicaçōes práticas do achado.
\end{abstract}

Palavras chaves: Doença de Chagas. Trypanosoma cruzi. Xenodiagnóstico.

O xenodiagnóstico ${ }^{5}$ constitui o método parasitológico indireto mais empregado para o diagnóstico da infeç̧ão chagásica crônica tanto no homem como em outros animais. Basicamente consiste no achado de formas infectantes do Trypanosoma cruzi nas fezes dos triatomíneos, a partir de 30 dias, aproximadamente, após terem sugado diretamente o sangue do animal. No homem a positividade no teste pode alcançar $49,3 \%^{16}$ ou até $68,5 \%^{6}$.

O método pode também ser realizado "in vitro" sendo denominado xenodiagnóstico artificial 31415 . Este também baseia-se na multiplicação do T. cruzi no triatomineo, mas lança-se mão, para a alimentação deste hematófago, de sangue previamente retirado de animal ou de paciente.

A necessidade de estabelecer com segurança o diagnóstico "post-mortem" da infecção chagásica levou-nos a empregar xenodiagnóstico artificial usando-se sangue de individuos necropsiados em diferentes periodos após o óbito.

\section{MATERIAL E MÉTODOS}

Em linhas gerais, foi utilizada a metodologia aplicada por Nussenzweig e $\mathrm{col}^{14}$ para o xenodiagnóstico artificial. $O$ sangue para alimentação dos triatomineos, foi retirado de 10 (dez) cadáveres de

1. Departamento de Patologia, Medicina Legal e Deontologia Médica da Faculdade de Medicina do Triângulo Mineiro, Uberaba, Minas Gerais.

2. Centro de Pesquisas René Rachou/FIOCRUZ, Belo Horizonte, Minas Gerais.

Financiado pelo Conselho Nacional de Desenvolvimento Científico e Tecnológico (PIDE V).

Endereço para correspondência: Dr. Edison Reis Lopes, Depto de Patologia, Medicina Legal e Deontologia Médica da Faculdade de Medicina do Triângulo Mineiro, 38100 Uberaba, Minas Gerais, Brasil.

Recebido para publicação em 25/3/1986. chagásicos crônicos com tempo de óbito variável de 2 a 14:30 horas (Tabela 1). Foi coletado logo após a retirada do coração ${ }^{8}$ precedida de punção do líquido pericárdico para os testes sorológicos. $O$ sangue coletado, cerca de 200 a $500 \mathrm{ml}$ foi transferido para um frasco vazio, esterilizado. A seguir, este sangue foi distribuido em placas de Petri, esterilizadas, de $10 \mathrm{~cm}$ de diâmetro, que após receberem o sangue, foram fechadas com luvas cirúrgicas.

A etapa seguinte consistiu em colocar essas placas em contato com outros frascos contendo triatomíneos. Estes últimos frascos tinham suporte de papelão, quadriculados que abrigavam os insetos da luminosidade mais intensa e serviam para que os insetos pudessem alcançar a fonte de alimento, isto é,o sangue contido nas placas de Petri. Cada frasco continha de 20 a 50 ninfas do 3. estágio de Triatoma infestans e Dipetalogaster maximus (Tabela 1). O conjunto, placas de Petri - frascos com triatomineos, foi levado à estufa a $37^{\circ} \mathrm{C}$ durante cerca de 60 minutos. Isto foi feito baseado no papel do termotropismo na alimentação dos hematófagos empregados ${ }^{13}$.

O diagnóstico da doença de Chagas nos cadáveres, dos quais se extraiu o sangue, foi feito baseado na positividade das reações (fixação de complemento, imunofluorescência e hemaglutinação) realizadas no líquido pericárdico ${ }^{10}$, bem como nos achados morfológicos macro e microscópicos do coração ${ }^{2}$.

Após 30 e 60 dias foi feita a pesquisa nas fezes obtidas por compressão abdominal dos triatomíneos individualmente, o que evitou sacrificá-los e com finalidade de posteriormente haver possibilidade de tentar-se o isolamento de cepas de $T$. cruz $i$.

\section{RFSULTADOS}

A Tabela 1 mostra os resultados com 30 e 60 dias após a realização do xenodiagnóstico, bem como os dados principais dos chagásicos necropsiados. $\mathrm{O}$ 
Lopes ER, Chapadeiro E, Brener Z, Franciscon JU, Cardoso JE, Adad SJ, Tostes Jr S. Xenodiagnóstico artificial "post-mortem" em chagásicos crònicos. Revista da Sociedade Brasileira de Medicina Tropical 19: 259-262, Out-Dez, 1986.

Tabela 1 - Xenodiagnóstico artificial em cadáveres de chagásicos crônicos

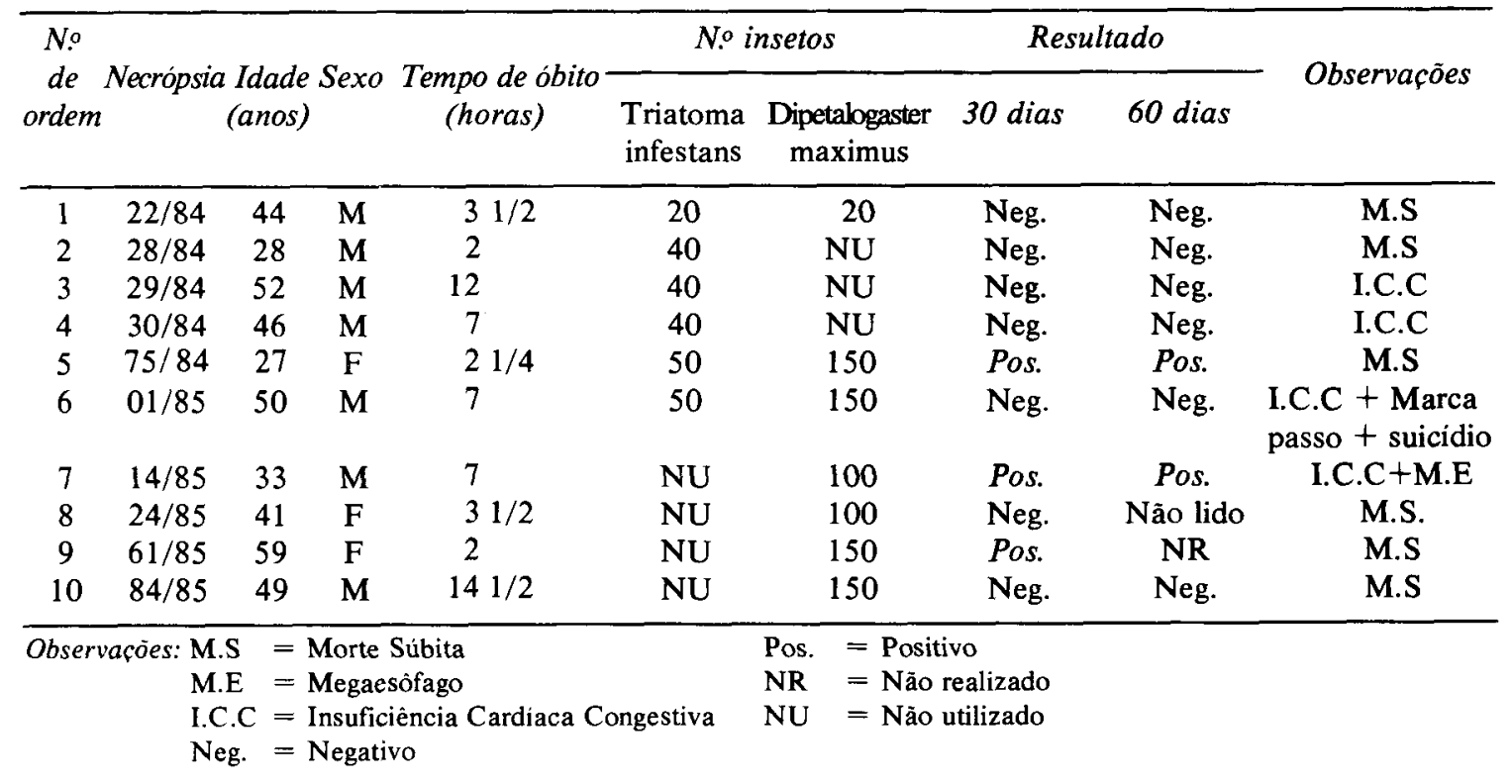

xenodiagnóstico foi positivo em 3 casos e todos ocorreram com $D$. maximus. A maioria dos triatomíneos empregados nos 10 (dez) testes foi capaz de sugar o sangue retirado dos cadáveres.

\section{DISCUSSĀO}

Conforme demonstram os presentes resultados em très (30\%) dos dez chagásicos necropsiados, nos quais aplicou-se o xenodiagnóstico artificial, havia $T$. cruzi no sangue que foi sugado pelos triatomineos utilizados no teste. Nos casos positivos, o tempo decorrido entre o êxito letal do hospedeiro até o inicio do repasto pelos triatomineos foi de respectivamente duas horas, duas horas e quinze minutos e sete horas. Estes resultados demonstram a possibilidade de dispor-se de mais de um método para o diagnóstico "post-mortem" da infeç̧ào chagásica.

A tanatosemiologia mostra-nos que os fenômenos cadavéricos iniciam-se em tempo variável após a morte. No sangue, a hemólise começa 2 a 3 horas após o óbito, mas há oxigênio disponivel no cadáver até várias horas após o óbito. Tanto isto é verdade que germens aeróbios como, por exemplo, o Bacillus subtilis, Proteus vulgaris e outros permanecem atuando até que se esgote o oxigênio disponivel. A partir dai germens anaeróbios, produtores de grande quantidade de gases, passam a agir e desencadeiam a putrefação o que não ocorreu nos nossos casos. Por outro lado, a queda da temperatura do hospedeiro, após a morte, pode favorecer a sobrevivência do parasita. No cadáver a temperatura sofre queda de 0,8 a $1^{\circ} \mathrm{C}$ por hora nas doze primeiras horas, o que nos leva a calcular que em nossos casos, no momento de necrópsia, os corpos parasitados pelo $T$. cruzi tinham temperaturas aproximadas entre 35 e $28^{\circ} \mathrm{C}$. Experimentalmente a temperatura afeta a infecção chagá$\mathrm{sica}^{4}$. Na temperatura de $35-37^{\circ} \mathrm{C}$ há clara redução da parasitemia e do número de parasitas intracelulares, diminuindo significativamente a mortalidade de animais inoculados aos passo que entre $10-18^{\circ} \mathrm{C}$ há aumento da virulência da infecção ${ }^{19}$. Vê-se portanto que não é de estranhar-se que $\circ T$. cruzi possa permanecer vivo até várias horas após a morte do corpo do hospedeiro.

Estes achados somados à metodologia já empregada para o diagnóstico "post-mortem" da doença de Chagas, tais como reaçōes sorológicas convencionais ${ }^{10} \mathrm{e}$ a lise mediada por complemento ${ }^{12}$ realizados no liquido pericárdico, constituem importante contribuição para o desenvolvimento das pesquisas em curso para um melhor conhecimento da doença de Chagas especialmente em sua forma indeterminada na qual, com freqüência, são escassas ou não se observam alterações morfológicas ${ }^{11}$.

Outra dedução prática de nossos achados aplica-se ao transplante de órgãos. Há relatos na literatura, de aquisição da infecção chagásica, no homem, via transplante de $\operatorname{rim}^{7}$ embora o órgão transplantado tenha sido retirado do doador vivo. Teoricamente os presentes dados, mostram que fato similar pode ocorrer mesmo com a retirada das vísceras após o óbito.

Nossos achados mostram também que há necessidade de precauções por parte daqueles que 


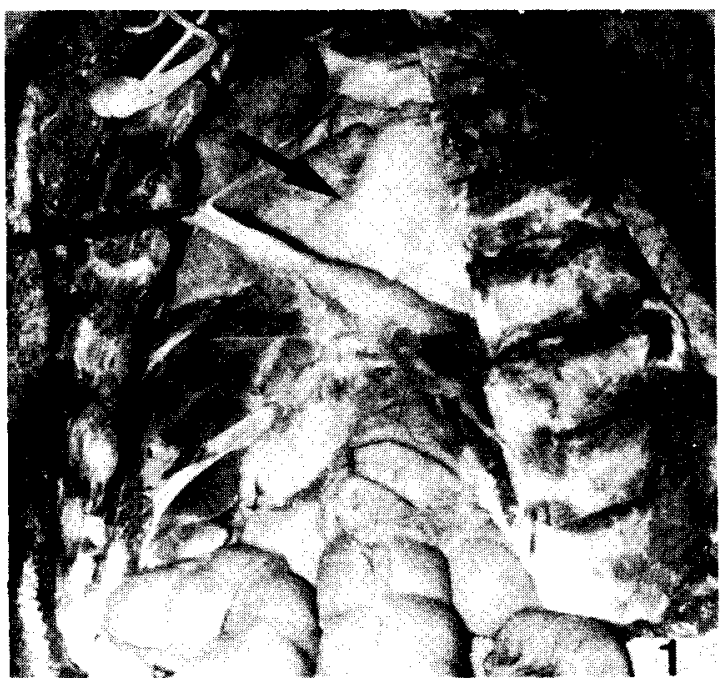

Figura 1 - Cavidade pericárdica aberta. A seta indica o coração "in situ".

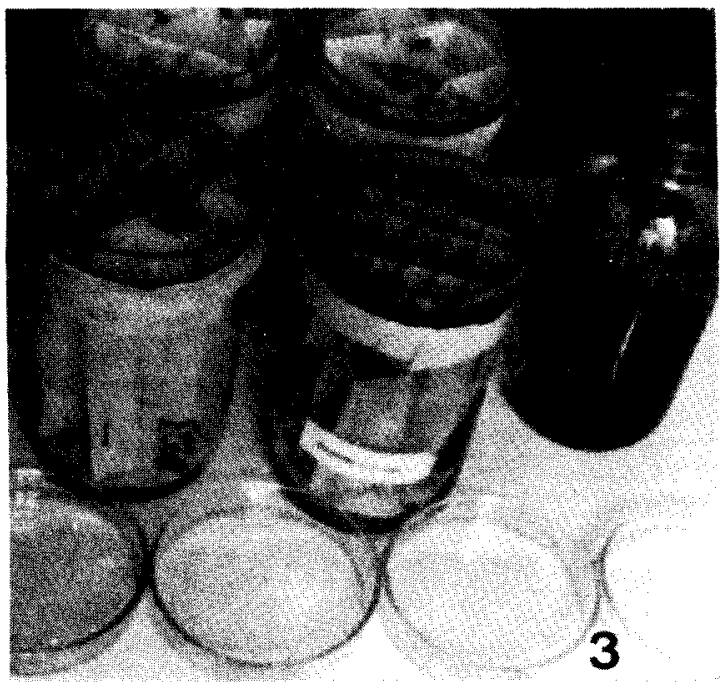

Figura 3 - Material utilizado para realização do xenodiagnóstico.

executam necropsias, uma vez que ha condiçòes de infeccāo pelo $T$. cruzi.

Finalmente, a possibilidade de obter-se xeno diagnosticos positivos após o obito, abre tambem novos campos de estudo da patologia da doença de C hagas. A perspectiva de isolamento e caracterizaçào do parasita que infecta o cadáver pode permitir estudos correlacionando achados anatomopatológicos com a cepa parasitária.

\section{SUMMARY}

Reliable post mortem diagnosis of Chagas' disease is highly desirable. Artificial xenodiagno-

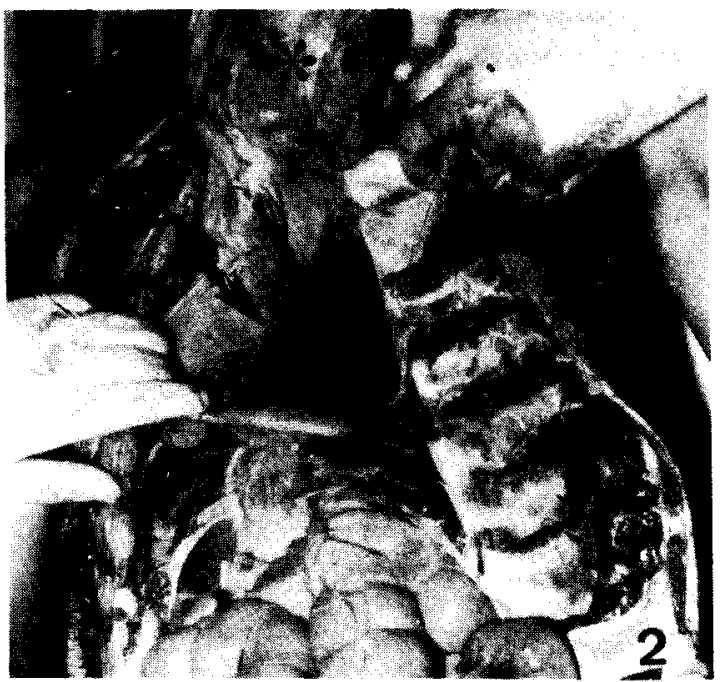

Figura 2 - Cavidade pericardica (seta) preenchida com sangue proveniente de secção da aorta. $O$ asterisco indica coraçào rebatido.

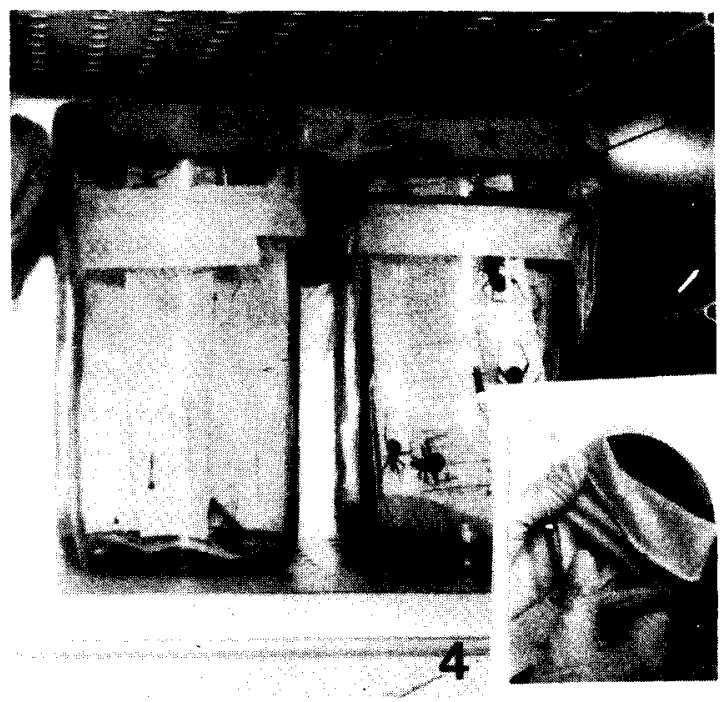

Figura 4 - Placas de Petri (asterisco) com sangue, aplicadas a frascos contendo triatomineos estando o conjunto dentro de estufa a $37^{\circ} \mathrm{C}$. No detalhe, placa de Petri contendo sangue, sendo fechada com luva cirurgica.

sis nas adapted for the human blood collected during autopsies in an attempt to reach that goal. The method was applied to ten proven chagasics. The test was positive in $3(30 \%)$ of the cases which were autopsied 2, $21 / 4$ and 7 hours after death. 
Lopes ER, Chapadeiro E, Brener Z, Franciscon JU, Cardoso JE, Adad SJ, Tostes Jr S. Xenodiagnóstico artificial "post mortem" em chagásicos crônicos. Revista da Sociedade Brasileira de Medicina Tropical 19: 259-262, Out-Dez, 1986.

The survival of Trypanosoma cruzi in a dead host and the practical use of the findings are discussed.

Key words: Chagas' disease. Trypanosoma cruzi. Xenodiagnosis.

\section{AGRADECIMENTOS}

Agradecemos aos senhores José Antônio Alves Filho e Marta Aparecida Camilo, pelo auxilio na parte técnica e a senhora Sueli de Melo, pela parte datilográfica.

\section{REFERÊNCIAS BIBLIOGRÁFICAS}

1. Bertelli MS. Influência da temperatura na diferenciação intracelular do Trypanosoma cruzi em cultura de tecido. Tese, Universidade Federal de Minas Gerais, Belo Horizonte, 1975.

2. Bogliolo L. Miocárdio, endocárdio, pericárdio. In: Bogliolo L (ed) Patologia, 3a edição, Guanabara-Koogan, 1982.

3. Borzone AR. Modificación de Berzone al método de Brumpt. In: Cornejo A. Enfermedad de Chagas-Mazza. Xenodiagnóstico. Semana Medica 56:181-183, 1949.

4. Brener Z. O parasita: relaçōes hospedeiro-parasito. In: Brener A, Andrade Z. (ed). Trypanosoma cruzi e Doença de Chagas, 1a edição, Guanabara-Koogan, 1979.

5. Brumpt E. O xenodiagnóstico. Aplicação ao diagnóstico de algumas infecçōes parasitárias e, em particular à tripanossomose de Chagas. Anais Paulistas de Medicina e Cirurgia 3:97-102, 1914.

6. Castro $\mathrm{CN}$. Influência da parasitemia no quadro clínico da doença de Chagas, Tese de Mestrado, Universidade de Brasilia, 1978.
7. Chocair PR, Sabaga E, Amato Neto V, Shiroma M, Goes GM. Transplante de rim: nova modalidade de transmissão da doença de Chagas. Revista do Instituto de Medicina Tropical de Sảo Paulo 23:280-182, 1981.

8. Franco EE. Manual Atlas de técnica de las autopsias, 1? edição, Salvat Editores SA. Barcelona, 1929.

9. Kolodny MH. The effect of envirommental temperature upon experimental trypanosomiasis (Trypanosoma cruzi) of rats. American Journal of Hygiene 32 (section C): $21-23,1940$.

10. Lopes ER, Chapadeiro E, Batista SM, Cunha Jr JG, Rocha A, Miziara L, Ribeiro JU, Patto RJ. "Postmortem" diagnosis of chronic Chagas' disease: comparative evaluation of three serological tests on pericardial fluid. Transactions of the Royal Society of Tropical Medicine and Hygiene 72: 244-246, 1978.

11. Lopes ER, Chapadeiro E, Andrade ZA, Almeida HO, Rocha A. Anatomia patológica de corações de chagásicos assintomáticos falecidos de modo violento. Memórias do Instituto Oswaldo Cruz 76:189-197, 1981.

12. Lopes ER, Pereira MES, Moraes C, Krettli AU, Brener Z. Anticorpos líticos detectados em liquido pericárdico de chagásicos crónicos. Revista da Sociedade Brasileira de Medicina Tropical 17:127-131, 1984.

13. Nicolle $P$, Mathis M. Le thermotropisme, facteur déterminant primordial pour la picure des Réduvide hématophages. Compte Rendu Societé de Biologie 135:25-27, 1941.

14. Nussenzweig V, Sonntag R. Xenodiagnóstico artificial. Novo Processo. Primeiros resultados positivos. Revista Paulista de Medicina 40:41-43, 1953.

15. Romaña C, Gil L. Xenodiagnóstico artificial. Anales del Instituto de Medicina Regional 2:57-60, 1947.

16. Schenone $\mathrm{H}$, Alfaro E, Rojas A. Bases y rendimiento del xenodiagnóstico en la infección chagásica humana. Boletin Chileno de Parasitologia 29:24-26, 1974. 